\title{
Peut-on gérer le couple coûts-valeur ?
}

\author{
Véronique MALLERET \\ Professeur Associé \\ Groupe HEC \\ F 78351 Jouy-en-Josas cedex \\ Tél. : 0139677202 \\ Fax. : 0139677086 \\ Email : malleret@hec.fr
}

Résumé : La communication conteste la possibilité de gérer conjointement les coûts et la valeur créée pour le client. Elle constate dans un premier temps qu'il n'existe pas de consensus sur la définition de la valeur. Elle montre ensuite que la notion de client n'étant pas homogène, la valeur pour le client sera au minimum variable et instable. Enfin, la communication étudie deux outils (le target costing et la méthode $\mathrm{ABC} / \mathrm{ABM}$ ) présentés par la littérature comme susceptibles de construire une relation entre les coûts et la valeur, et elle montre que ces outils n'établissent que de façon très imparfaite un lien entre les attributs du produit qui sont à l'origine de la valeur et les coûts mobilisés pour produire ces attributs.

MotS CLES : COUT, VALEUR, COUT CIBLE, ABC/M. 


\section{PeUt-ON Gerer LE COUPLE COUTS-VAleur ?}

\section{INTRODUCTION}

Depuis environ deux décennies, les spécialistes de la comptabilité de gestion ont pris conscience du fait que le contrôle des coûts ne suffisait pas pour assurer le succès économique durable de l'entreprise, l'accumulation des coûts au long d'un processus de production n'entraînant pas nécessairement la génération d'un chiffre d'affaires au moins équivalent. En particulier, durant les années 80 , les industriels et les spécialistes de la gestion des opérations se sont efforcés d'éliminer les «opérations sans valeur ajoutée », comme le stockage ou les contrôles qualité, opérations qui avaient précisément comme caractéristique d'augmenter les coûts sans augmenter la valeur pour le client.

Cette prise de conscience a été suivie par une mobilisation générale visant à développer des outils destinés à gérer conjointement les coûts et la valeur, à les mettre en relation et à effectuer des arbitrages entre ces deux catégories d'éléments : «L'enjeu est d'optimiser l'offre de l'entreprise, en adaptant les coûts qu'elle engage à la valeur que son produit représente pour le client» (Bouquin, 2004, p. 139). Parmi les tentatives les plus connues, on peut citer le coût cible et le management des activités et des processus. Une certaine ambition s'observe dans ce domaine comme on peut le constater à la lecture de quelques titres d'articles ou de communications : «Le déploiement de la valeur par les processus » (Lorino, 1995a), "Simultaneaously managing cost and value: The challenge » (Lebas, Mévellec, 1999), «Cost mangement and value cration: the missing link» (McNair et al. 2001).

Mais les outils cités, les méthodes proposées permettent-ils réellement de gérer les coûts et la valeur ? Peut-on mesurer la valeur pour le client, la gérer et ensuite la relier aux coûts générés par les activités internes d'une organisation dans une perspective de management de la performance? C'est à ces questions que nous allons nous efforcer de répondre dans cette communication.

Dans une première partie, nous essaierons de définir ce qu'est la valeur pour le client; dans un premier temps nous essaierons de définir la notion de valeur et, en particulier, nous essaierons de voir si elle se distingue du prix payé par le client. Puis, nous montrerons en quoi la notion de client est complexe et que les clients sont en réalité «multiples, instables et volatiles » ce qui rend délicates ou à tout le moins relatives la mesure et la hiérarchisation des 
valeurs qu'ils perçoivent. Nous illustrerons cette première partie par l'exemple du développement du système PAX chez Michelin.

Dans la seconde partie de la communication, nous montrerons en quoi c'est la relation entre coûts et valeur qui est problématique. Pour ce faire, nous analyserons les principaux outils proposés en comptabilité de gestion pour construire cette relation, à savoir la méthode des coûts cibles et les méthodes $\mathrm{ABC} / \mathrm{ABM}$ et nous montrerons leurs limites théoriques et pratiques.

\section{LA VALEUR POUR LE CLIENT}

Au cours des deux dernières décennies, le terme de valeur s'est imposé de plus en plus fréquemment, en particulier avec l'insistance portée sur la création de valeur pour l'actionnaire (Ittner et Larcker, 1998). Toutefois, la popularité du terme de valeur ne s'est pas accompagnée d'une clarification de sa définition et des ambiguïtés persistent, en particulier lorsque l'on emploie le mot valeur sans préciser s'il s'agit de valeur pour l'actionnaire ou de valeur pour d'autres parties prenantes: salariés, clients, fournisseurs, collectivités (Bourguignon, 2005).

Laissant de côté la valeur pour l'actionnaire et le débat sur une possible convergence des valeurs pour les différentes parties prenantes de l'entreprise, nous nous concentrons pour notre part sur la valeur pour le client. En effet, c'est à cette dernière que se réfèrent les chercheurs spécialisés en comptabilité de gestion quand ils se proposent de mettre en relation les coûts engendrés par des clients ou des produits et la valeur créée par l'entreprise pour ces mêmes clients, dans une perspective de management de la performance. ${ }^{1}$

Dans cette première partie, nous allons tenter de définir la valeur pour le client en nous appuyant sur les définitions de la valeur données par les économistes et en rapprochant les notions de valeur et de prix. Puis nous montrerons que la multiplicité des clients, l'instabilité de leurs préférences rendent complexe et problématique la mesure de la valeur pour des clients.

\footnotetext{
${ }^{1}$ Nous utiliserons dans ce travail le terme de « valeur pour le client » afin de distinguer celle-ci de la valeur du client pour l'entreprise qui se calcule en actualisant les profits futurs d'un client sur sa durée de vie.
} 


\subsection{QU'EST CE QUE LA VALEUR?}

\subsubsection{Valeur d'échange, valeur d'usage ou valeur-utilité ?}

Les définitions du mot valeur peuvent être recherchées dans trois champs principaux : les sciences et les arts, l'économie et la philosophie. Nous ne nous attarderons pas sur les définitions issues des sciences, des arts et de la philosophie (qui ont été rappelées par Bourguignon, 2005). Nous allons en revanche considérer les définitions issues de l'économie car elles peuvent nous aider à mieux comprendre ce qu'est la valeur d'un bien ou d'un service pour le client.

Deux conceptions de la valeur, la valeur d'usage et la valeur d'échange, sont présentes dans les travaux de Smith dès 1776 : «Le mot valeur a deux significations différentes ; quelquefois il signifie l'utilité d'un objet particulier et quelquefois, il signifie la faculté que donne la possession de cet objet d'acheter d'autre marchandises »(Smith, 1991, cité par Simon, 2000). Ramirez (1999) a proposé une étude historique du mot valeur dans la langue française dont il ressort que le sens le plus ancien (900 ans) du mot valeur se réfère à l'adaptation à un usage tandis que le sens de mesure du mot valeur, utilisé dans le commerce, ne serait apparu que 200 ans plus tard, fondant ainsi la valeur-échange.

A la suite des travaux de Smith, l'économie distingue la valeur d'échange et la valeur d'usage :

- La valeur d'échange est le «taux auquel une marchandise s'échange contre une autre marchandise. Synonyme de prix relatif. » (Echaudemaison, 1989, p. 456)

- La valeur d'usage est «l'utilité d'un bien évaluée soit de manière objective et générale (le pain fournit un certain nombre de calories), soit de manière subjective et donc variable d'un individu à l'autre. La valeur d'usage est relative au besoin, la valeur d'échange relative à un autre bien. » (Echaudemaison, 1989, p. 456)

Pour les économistes classiques la valeur d'usage est égale à la somme des coûts nécessaires pour produire le bien (ou pour Marx, au coût du travail engagé) tandis que pour les néoclassiques, cette valeur d'usage est appelée valeur-utilité (Simon, 2000, Roche, 2002) et se définit par rapport aux besoins de l'utilisateur.

La première question que l'on peut se poser, lorsque l'on parle de gestion du couple coûtsvaleur pour le client, est de savoir si nous nous référons à la valeur d'échange, à la valeur d'usage ou à son avatar néo-classique, la valeur-utilité. En effet, si l'on retient comme définition de la valeur la valeur d'usage au sens des économistes classiques, on peut 
considérer que les coûts sont identiques à la valeur et l'intérêt de la question de recherche tombe de lui-même. On est alors dans la logique des systèmes de coûts antérieurs aux années 90 dans lesquels l'hypothèse était faite que l'addition de coûts conduirait à une valeur que le client ne pourrait que reconnaître (Mévellec, 2000a, p. 34). Il semble donc, si nous voulons que notre question ait un sens, qu'il faille retenir soit la valeur-utilité soit la valeur d'échange pour définir la valeur pour le client.

L'adoption de la valeur-utilité (par opposition à une valeur fondée sur les coûts ou sur la quantité de travail) introduit d'emblée le fait que la valeur d'un bien dépend du contexte dans lequel se trouve l'utilisateur. Comme le mentionne Simon (2000) en s'appuyant sur les travaux de Perroux, « elle conduit à considérer la valeur non pas comme une réalité objective reposant sur des entités (le fer, le blé, la société...) dans une économie figée mais à la fonder sur des comportements humains. La valeur devient ainsi une donnée subjective et contingente. » (p. 1252)

La valeur pour le client a donc un caractère relatif, d'une part parce qu'elle dépend des caractéristiques du consommateur et de la situation dans laquelle il se trouve et d'autre part parce que le consommateur va apprécier la valeur d'une offre par comparaison avec les offres de concurrents :

- «La valeur économique offerte au consommateur... est la valeur relative qu'un produit donné offre à un consommateur dans une application particulière. C'est le montant maximum qu'il serait disposé à payer, en supposant qu'il est parfaitement informé concernant le produit et les offres des concurrents. » (Teller, 1999, p. 235)

- «Après comparaison des différentes offres, le client choisira celle dont il pense qu'elle lui en donne pour son argent. La valeur est bien ici une notion relative puisqu'elle résulte d'une comparaison avec l'offre de la concurrence. » (Barwise et Meehan, 1999)

Cependant, comme on peut le constater en lisant attentivement les deux citations qui précèdent, reconnaître le caractère relatif de la valeur ne suffit pas pour autant à adopter une perspective de valeur d'utilité. La référence au prix, et donc à la valeur d'échange, est bien présente. Ceci va nous conduire dans un deuxième temps à nous interroger sur les relations qui lient la valeur pour le client au prix. 


\subsubsection{Valeur ou prix ?}

Il semble qu'il existe une opposition entre la valeur d'échange, qui s'exprimerait en termes monétaires (et pourrait s'assimiler au prix) et la valeur-utilité qui s'exprimerait en utilité, en satisfaction, «le bien ou le service en question venant remplir certains des besoins de l'utilisateur » (Bourguignon, 2005). D'où la question : la valeur est-elle assimilable au prix ou est-elle distincte de ce dernier ? Plus précisément, la valeur d'un produit ou d'un service peutelle être approchée par son prix ou même identifiée à son prix ? Ou bien est-elle autre chose dont le prix ne serait qu'un des éléments constitutifs ou explicatifs, l'un des attributs ? Nous avons essayé de structurer les réponses apportées à cette question en distinguant les auteurs qui assimilent la valeur au prix et ceux qui considèrent que le prix n'est qu'un des éléments explicatifs de la valeur ; cependant, beaucoup d'auteurs adoptent une position ambiguë comme on va le voir maintenant.

\section{Le prix, approximation de la valeur}

Pour certains auteurs, que l'on pourrait considérer comme les tenants de la valeur d'échange, la valeur pour le client est le prix que celui-ci est prêt à payer pour acquérir un bien ou un service; le prix est donc une approximation, un indicateur de la valeur d'un bien pour le client.

- Dans son article sur valeur et comptabilité, Simon (2000) pose en introduction que «le prix - constaté lors d'une transaction sur un marché - est l'expression d'une valeur ; ou inversement, que la valeur d'un bien constitue le fondement de son prix. » (p. 1245)

- Pour Lorino, (1995b, p. 126) « le prix, comme tout indicateur chiffré, peut constituer un signe de valeur mais il n'est pas la valeur ». De même, pour McNair et al. (2001), le prix de marché représente un proxy de la valeur actuelle nette que le client va retirer du produit ou du service acheté. » (p. 33)

- Dans son article sur la relation entre coût et valeur, Mévellec part de l'idée que « le prix est, dans l'échange, la cristallisation de la valeur» (2000a, p. 32) et parle de «la valeur que les clients vont accepter de payer. » (p. 33)

Si le prix est l'expression de la valeur d'un produit ou d'un service, l'origine de cette valeur, $\mathrm{du}$ fait que les clients sont prêts à payer se trouve dans les attributs ${ }^{2}$ du produit/service.

2 La notion d'attributs peut être rapprochée de la notion de fonctions issue de la tradition d'analyse de la valeur industrielle, sans cependant la recouper tout à fait. Lorino parle le plus souvent de fonctionnalités tandis que les spécialistes de marketing emploient davantage le terme de bénéfices. 
Bromwich (1990) a précisé la notion d'attributs, soulignant que l'attraction d'un produit sur le consommateur n'est pas le fait du produit lui-même, de façon monolithique, mais d'un ensemble de caractéristiques : «Ici, les produits sont vus comme constitués d'un ensemble d'attributs objectifs ou de caractéristiques qu'ils offrent aux clients... Ce sont ces attributs qui attirent réellement les clients... Ces attributs peuvent inclure divers éléments liés à la qualité, comme des performances opérationnelles, la fiabilité, les conditions de garantie, des aspects physiques comme le niveau de finition, et des éléments de service comme la sécurité d'approvisionnement ou le service après-vente. » (p. 30). Cette vision est confirmée par McNair et al. (2001, p. 36) : «Une abondante littérature de marketing démontre que les clients achètent un bouquet d'attributs de produits et services quand ils font un choix sur un marché ». Mévellec (2005) considère également que la source de la valeur réside dans les attributs lorsqu'il affirme : «La valeur ... est le résultat d'un assemblage de fonctionnalités perçues par le client et chacune porteuse d'une dimension utile » (p. 57)

La première option qui s'offre consiste donc à approcher la valeur par le prix du produit/service. Cette option suscite quelques interrogations.

1. Il faut d'abord souligner que les différentes propositions que nous avons citées ne sont pas parfaitement identiques. En particulier, le «prix que le client est prêt à payer » n'est sans doute pas égal au prix de marché, ni au prix que le client va payer finalement. En effet, entre ce que le client est prêt à payer et ce qu'il paie, il y a la capacité de l'entreprise à transformer une valeur créée en valeur acquise, capacité qui dépend de la situation concurrentielle, du rapport de force entre acteurs, des caractéristiques du marché, en particulier dans les activités de type B to B (Kœnig, 2004, p.230). De fait, Charreaux et Desbrières (1998) définissent précisément ainsi la valeur pour le client : «Il y a valeur créée si le client obtient le produit à un prix inférieur à son prix d'opportunité, c'est à dire le prix qu'il aurait été disposé à payer. » (p. 61)

2. Concrètement, si nos systèmes de comptabilité de gestion gardent trace facilement du prix payé par les clients et du chiffre d'affaires qu'ils génèrent, il est plus difficile de se faire une idée du prix de marché et encore plus du prix que client est (était) prêt à payer. Dans ces conditions, est-il possible de fonder la mesure de la valeur pour le client par le prix qu'il est « prêt à payer »? 
3. Enfin, cette première option, assimiler la valeur au prix, pose une question annexe : si la valeur pour le client peut être assimilée au prix qu'il va payer, pourquoi alors parler de valeur pour le client et non de prix ou de chiffre d'affaires ? Y a-t-il une différence entre la gestion du couple coûts-valeur et la traditionnelle gestion du couple coûtschiffre d'affaires par les marges ? Ou s'agit-il seulement de remplacer le mot chiffre d'affaires par le mot valeur afin de donner au client l'impression qu'on lui apporte quelque chose plutôt qu'on ne lui «prend son argent »?

\section{Le prix, attribut de la valeur}

A l'opposé, d'autres auteurs considèrent que le prix est un des attributs, une composante de la valeur mais distincte de celle-ci. Dans ce cas, la valeur serait une synthèse des différentes caractéristiques du bien, le prix étant une caractéristique ou un attribut parmi d'autres.

C'est la position retenue par Aurier et al. (2004) qui distinguent la valeur globale d'un produit et sa valeur de consommation. Reprenant des définitions antérieures issues de la littérature de marketing, ils mentionnent que « la valeur globale d'un produit ... résulte de la confrontation entre les bénéfices et les sacrifices associés à la consommation. Elle est définie comme l'évaluation globale de l'utilité d'un produit fondée sur les perceptions de ce qui est reçu et donné ou comme le rapport entre les bénéfices et les sacrifices perçus » (p. 2). A l'opposé, le travail sur ce que ces auteurs appellent la «valeur de consommation » est analytique et s'intéresse aux éléments qui sont à l'origine de la valeur globale. L'article propose une liste générique de ces éléments qui se situent à un niveau de généralité et d'abstraction supérieur à ce que nous appelons attributs dans la littérature de comptabilité de gestion ${ }^{3}$.

De la même façon, pour Barwise et Meehan (1999), c'est le rapport valeur perçue/ prix qui détermine le comportement du consommateur : «... la perception de valeur par le client, c'està-dire l'appréciation qu'a le client de l'ensemble des avantages retirés d'un produit ou d'un service par rapport au coût total représenté par son prix d'achat ou sa jouissance. Même s'ils ont généralement d'autres priorités, les consommateurs ont pratiquement tous en tête une notion implicite du «bon » rapport qualité-prix. Après comparaison des différentes offres, le client choisira celle dont il pense qu'elle lui en donne pour son argent. »

Jallat (2002) est particulièrement explicite puisque, pour lui, c'est justement l'écart entre la valeur perçue par le client et le prix qui fonde la compétitivité de l'entreprise : «A ce titre la différence entre le prix de vente d'un produit et sa valeur perçue par le consommateur doit

\footnotetext{
${ }^{3}$ Ce niveau d'abstraction pourrait poser un problème par la suite si nous voulions utiliser ces facteurs explicatifs de valeur pour les relier à des activités et à des coûts dans un modèle destiné à gérer la performance.
} 
être interprété comme... un vecteur de compétitivité marginale qui doit être maintenu, capitalisé et développé par l'entreprise. (2002, p. 70)

Porter (1985, p. 3) est plus ambigu en affirmant : « La valeur est ce que les clients sont prêts à payer » mais en ajoutant immédiatement : « ...une valeur supérieure vient du fait d'offrir des bénéfices équivalents à ceux des concurrents pour des prix plus bas ou de fournir des bénéfices uniques qui font plus que contrebalancer l'accroissement du prix ».

Enfin, Lorino indique que «la valeur apparaît à travers ce jugement d'opportunité sur le rapport entre fonctionnalités et prix. »(Lorino, 1995b, p. 127). Il précise en 1997 : «La valeur est le jugement porté par la société (notamment le marché et les clients potentiels) sur l'utilité des prestations offertes par l'entreprise comme réponse à des besoins. Ce jugement se concrétise par des prix de vente, des quantités vendues, des parts de marché, des revenus, une image de qualité, une réputation, etc. » (p.67)

Les auteurs qui adoptent la position selon laquelle la prix est une des composantes de la valeur sont en général proches de la stratégie et du marketing; leur approche de la valeur présente de plus les caractéristiques suivantes :

- La valeur à laquelle ils se réfèrent est une valeur perçue par le client : elle n'existe pas en tant que telle. Il s'agit d'une appréciation (Barwise et Meehan, 1999), de représentations mentales (Jallat, 2002) des clients qui dépendent elles-mêmes de leurs attentes.

- La valeur se définit par rapport aux offres des concurrents. Ainsi, la valeur perçue d'un produit/ service peut diminuer sans qu'aucun de ses attributs ne soit modifié, simplement parce qu'un concurrent a modifié son offre. La valeur est donc éminemment relative.

\subsubsection{La valeur : donnée, construite ou interprétée?}

Un point commun à l'ensemble des auteurs étudiés apparaît cependant : que la valeur puisse être approchée par le prix ou que celui-ci soit une de ses composantes, la valeur est externe à l'entreprise ; dans le premier cas, elle est déterminée par un marché, dans le deuxième cas par les préférences d'un client, mais dans les deux cas, elle est constatée et non construite par l'entreprise. C'est donc une information qu'il faudra aller chercher à l'extérieur et qui ne peut être calculée en interne, contrairement aux coûts.

Cependant, le fait que la valeur soit une information émise par le marché est lui-même contesté par certains auteurs qui mettent en doute l'existence du marché en tant que tel, qui 
serait doté d'une vie autonome, indépendante des acteurs qui le composent. Pour Bréchet et Desrumeaux (2001), la valeur est autant une donnée qui s'impose à l'entreprise de l'extérieur qu'une construction par des acteurs (individus, organisations) qui interviennent dans la façon dont les marchés disent la valeur, et qui essaient de façonner les marchés par des règles du jeu, des priorités : «La valeur, considérée du point de vue des organisations, est une réalité à la fois donnée et construite et pour le moins autant construite par les acteurs, et notamment par les organisations, que par les marchés. » (p. 209)

Pour Lorino, la valeur est en fait une interprétation. Selon lui, les «produits ont de la valeur s'ils offrent une ou plusieurs fonctionnalités, en désignant par fonctionnalités l'aptitude à un besoin ressenti par un groupe social »(1995b, p. 125). La question de savoir si certains produits apportent plus de valeur que d'autres ou s'ils consomment plus de ressources qu'ils ne créent de valeur est «affaire de jugement, l'affaire d'une interprétation complexe sur l'utilité relative de différents types de fonctionnalités pour divers groupes sociaux. » (1995b, p. 126)

Cette notion d'interprétation rejoint l'idée émise par Gadrey en 1988. En effet, pour celui-ci, l'output, la production d'un service ne sont pas définis dans l'absolu ou en se référant aux caractéristiques physiques de «ce qui sort » du système productif. Par exemple, l'Education Nationale et une entreprise privée de formation continue, bien qu'exerçant des activités similaires, n'auront pas les mêmes mesures d'output, ne seront pas jugées sur les mêmes critères en termes de création de valeur. En réalité, la définition des outputs dépend de l'environnement social, des acteurs et de leurs systèmes de valeurs : «Tout concourt à démontrer, que, pour de tels services, ce sont les rapports économiques et les règles sociales et institutionnelles qui décident de ce qu'est le résultat essentiel du service, son output principal. » (p. 133). Ainsi, la valeur qu'un client attribue à un bien ou à un service serait liée à l'environnement socio-économique de ce dernier, à son « système de valeurs ». 


\section{Conclusion}

Il est difficile de conclure et de proposer une définition de la valeur à partir d'éléments aussi peu clairs et souvent contradictoires. A l'issue de cette première partie, deux options s'offrent à nous :

- Reconnaître que la valeur pour le client est une notion mal définie, qui ne suscite pas de consensus. Il faut alors en conclure que gérer la valeur (et les coûts avec elle) est quasiment une illusion, à moins que l'on ne puisse mesurer et gérer quelque chose qui n'est pas défini précisément.

- Adopter une définition imparfaite, certes, mais susceptible de nous permettre d'avancer et de répondre à une interrogation légitime des praticiens : si les coûts et la valeur sont deux notions disjointes, il faut bien les appréhender pour les gérer.

C'est pourquoi, pour poursuivre ce travail, nous retiendrons que la valeur est une appréciation de l'utilité globale d'un produit ou d'un service par un client; nous admettrons que, pour des motifs opérationnels, cette utilité peut parfois être approchée par le prix que le client paie pour se procurer ce produit ou ce service, mais en restant conscient du fait que ce prix n'est qu'un indicateur de la valeur.

\subsection{QUEL CLIENT?}

Nous nous sommes d'abord intéressé à la notion de valeur pour le client et nous avons vu à quel point il était difficile de définir le terme de valeur. Nous allons maintenant nous pencher sur le deuxième élément du problème, le client, et nous allons montrer que celui-ci n'est pas monolithique et cohérent mais multiple et changeant. De ce fait, définir un panier d'attributs auxquels le client est sensible et établir une hiérarchie entre ces attributs va se révéler délicat comme le mentionnent Bréchet et Desrumeaux (2001) : «Du côté de la demande, apprécier ce qui vaut pour le client et l'utilisateur et le concrétiser à travers un panier d'attributs dont il pourra disposer est pour le moins délicat, voire bien souvent relève du pari et de l'expérimentation.» (p. 240). Pour Mévellec également (2000b), « la valeur est une notion floue tant en raison des fluctuations des comportements des clients que de la difficulté à interpréter les signaux reçus de l'environnement » (p. 398). Au minimum il faudra considérer que l'établissement d'une liste d'attributs et de leurs poids relatif est en général une tâche complexe qui conduit à des résultats instables pour des raisons que nous allons maintenant détailler. 


\subsubsection{Du client aux segments de clients}

Tout d'abord, sauf dans quelques exceptions d'une activité B to B où l'on traite les clients un par un, les clients sont nombreux et ne forment pas un ensemble homogène. Au minimum, il y a des segments de clients. La liste des attributs significatifs pour chacun des segments n'est pas nécessairement la même pour tous les clients et l'importance que chaque segment de clients accorde aux différents attributs n'est pas la même non plus.

Dans une interview à Marketing Magazine en 1997, Dubois retrace le chemin parcouru en ce sens par le marketing : «Nous montrons l'évolution qu'il y a eu d'un marketing de masse jusqu'à un marketing par segment, un marketing de niche puis un marketing individuel.» (p. 43)

\subsubsection{Du client au client situationnel}

Si l'on change d'échelle d'analyse et que l'on se place au niveau d'un seul client, il peut arriver que la liste des attributs et les échelles de préférence de ce dernier ne soient pas stables dans le temps. Les préférences et le comportement d'un client dépendent de sa situation, ce qui a donné naissance à un courant de marketing appelé marketing situationnel «par ce qu'il ne se construit plus à partir des caractéristiques des produits ou des consommateurs, mais à partir de situations dans lesquelles ceux-ci rencontrent ceux-là. » (Dubois, 1996, p. 13).

Pour Dubois, le consommateur est devenu un caméléon : «Adepte de la première classe lorsqu'il voyage en note de frais, il négocie pied à pied avec Nouvelles Frontières le prix de ses vacances ; attentif à la liste d'achats lorsqu'il les effectue seul, il laisse ses enfants remplir son chariot dès que ceux-ci l'accompagnent. Tout comme le caméléon n'est ni vert, ni rouge, ni jaune mais peut être effectivement tout cela, le consommateur d'aujourd'hui n'est de façon permanente, sensible ni à la marque, ni aux prix, ni à la qualité.» (1996, p. 13). Et Dubois ajoute en 1997 : «Aujourd'hui, il est peut-être plus intéressant de faire des typologies de situations que de consommateurs. » (p. 44)

Pratiquement, ceci implique que l'entreprise ne doit pas nécessairement proposer des attributs et des niveaux d'attributs constants à ses clients (par exemple il faudrait traiter avec plus d'attention un client qui vient d'attendre une demi-heure qu'un client qui n'a pas attendu). D'un point de vue plus théorique, on peut en déduire que la liste d'attributs déterminant la valeur n'est pas stable, pas plus que le poids relatif des attributs; ils dépendent de la situation ou du mode de consommation dans lesquels se trouvent les clients. 


\subsubsection{Des clients aux quasi-clients}

La notion de valeur pour l'actionnaire a été élargie à la notion de valeur pour le client et même à celle de valeur partenariale (Charreaux et Desbrières, 1998). Ceci permet d'accroître le nombre et le type de parties prenantes de la valeur créée. Cependant, la plupart des auteurs définissent les parties prenantes en s'inspirant de la chaîne de valeur de Porter et mettent en avant les acteurs que celui-ci mentionne (clients, salariés, fournisseurs) sans chercher à examiner de façon plus fine chacune de ces catégories.

Or, les organisations n'ont pas seulement des clients, des segments de clients ou des parties prenantes traditionnelles. Elles ont des catégories de clients différentes, que nous appellerons quasi-clients. Ceux-ci peuvent avoir des listes d'attributs différentes ou dotées de valeurs différentes. Par exemple, si le premier client de l'hôpital est le malade lui-même, on peut considérer que plusieurs catégories de «quasi-clients" gravitent autour de l'hôpital : la famille du malade, le médecin de ville qui y a envoyé son patient, les étudiants en médecine qui y font leurs études. Comment dans ce cas définir la valeur du produit « hôpital »? S'agitil d'un agrégat des préférences des différentes catégories de quasi-clients, certains quasiclients sont-ils privilégiés et «sur-valorisés» au détriment des autres ? De même, dans une école de gestion qui est le client et qui va apprécier la valeur produite ? L'étudiant qui «consomme le produit», ses parents qui paient ses études, les entreprises qui vont le recruter? Qui va définir la liste des attributs et leur valeur relative?

\subsubsection{De l'organisation cliente aux clients}

Enfin, en particulier dans les marchés de type B to B, derrière le terme de clients se cachent souvent plusieurs acteurs au sein d'une même organisation. Ainsi, dans les marchés de biens d'équipement, le fournisseur a fréquemment plusieurs interlocuteurs : l'usine qui va acquérir l'équipement (et qui va se focaliser sans doute sur le coût de la pièce produite), le service achats de l'entreprise qui sera plus sensible au prix d'achat ou au fait d'homogénéiser le parc de machines entre sites, l'opérateur qui utilisera la machine et sera plus attentif à sa fonctionnalité. A nouveau, se pose la question de savoir qui est le client et sur quelles préférences l'entreprise va construire une liste d'attributs permettant de déterminer la valeur de son offre.

D'une façon plus générale, peut-on traiter de la même façon et avec les mêmes cadres conceptuels la valeur pour le client dans un contexte B to $\mathrm{B}$, dans lequel le client va effectivement essayer de calculer économiquement ce que peut lui rapporter un produit ou un 
équipement, et dans un contexte $\mathrm{B}$ to $\mathrm{C}$, avec un consommateur final individuel, qui fonde peut-être moins ses préférences sur une rationalité économique?

\subsubsection{De la valeur créée pour le client à la valeur perçue par le client}

Enfin, de nombreux travaux de marketing montrent que la valeur créée telle qu'elle est perçue par l'entreprise est en général différente de la valeur créée telle qu'elle est perçue par le client (Carmon et Ariely, 2000).

Plusieurs phénomènes vont venir s'intercaler entre la valeur telle qu'elle est produite par l'entreprise (en quelque sorte les attributs objectifs du produit ou du service) et la valeur perçue par le client : c'est le cas de la communication sur le service qui peut avoir inutilement augmenté les attentes en «survendant» le service ou des expériences antérieures qui font que le client modifie ses attentes, la valeur des attributs et ses perceptions. Ainsi, un client qui a eu du mal à stationner sa voiture pour visiter un parc d'attraction un jour de forte affluence aura tendance à sur-valoriser l'attribut «facilités de stationnement» dans une enquête alors que, s'il avait expérimenté le même service un jour de faible affluence, il aurait peut-être survalorisé le critère « animation-ambiance ».

La perception de valeur par le client est une donnée relative qui dépend aussi d'un certain nombre de paramètres qui ne sont pas nécessairement contrôlés et contrôlables par l'entreprise. Ainsi, la perception de valeur peut aussi dépendre des autres clients (qui dérangent le client ou dégradent l'image qu'il se faisait d'un produit de luxe). Elle peut aussi dépendre de sous-traitants, de fournisseurs plus ou moins bien maitrisés.

Enfin, dans tous les cas où le client contribue à la réalisation du produit ou du service qu'il consomme, la façon dont il va juger sa contribution peut modifier la perception qu'il a du produit et de la valeur créée. En cas d'incident par exemple, va-t-il avoir tendance à s'attribuer la responsabilité de l'incident et s'estimer satisfait malgré l'incident ou au contraire va-t-il rendre l'entreprise responsable des problèmes rencontrés ? ${ }^{4}$

Pour ces différentes raisons, il est tout à fait probable qu'il existe un écart entre la valeur produite par l'entreprise et la valeur perçue par les clients. C'est pourquoi, il est important, dans une perspective de gestion de la performance, de savoir si la valeur que le système de gestion souhaite relier aux coûts est la valeur créée ou si c'est la valeur perçue (qui est, en principe, la seule capable de modifier la propension à payer du client).

\footnotetext{
${ }^{4}$ Des travaux de marketing ont montré par exemple que la propension à réclamer des clients était très différente selon le degré de responsabilité qu'ils s'attribuaient dans l'échec. (Snellman et Vihtkari, 2003)
} 


\subsection{UN EXEMPLE DE RECHERCHE DE LA VALEUR CLIENT : LE SYSTEME PAX DE MICHELIN ${ }^{5}$}

Au cours des années 90, Michelin a développé un système innovant comprenant une roue et un pneu auquel l'entreprise a donné le nom de système PAX. Le système PAX présente les avantages suivants ${ }^{6}$ :

- Une meilleure tenue de route grâce à des flancs plus courts, ce qui donne une meilleure réactivité du véhicule ;

- Un pneu indéjantable, donc plus de sécurité ;

- La possibilité de rouler à plat sur au moins 200 kilomètres à 80 km/h.

Pour évaluer l'intérêt économique de ce nouveau système et gérer le projet, l'entreprise a développé un certain nombre d'outils destinés à mesurer la valeur créée pour le client par cette innovation. Un certain nombre d'enseignements peuvent en être tirés qui viennent pour partie confirmer l'analyse théorique que nous avons menée jusqu'ici.

\section{La notion de clients est multiple}

Il y au minimum deux types de clients pour lesquels la notion de valeur a un sens dans ce cas :

- Le client final, acheteur de la voiture, qui va par exemple être sensible au fait que sa voiture ne crève plus, ou que, si elle crève, elle n'est plus immobilisée. Le client final est également sensible à des attributs concernant l'usure des pneus, etc.

- Le constructeur automobile qui va intégrer l'innovation dans ses véhicules. Ce constructeur va pouvoir vendre au client final l'attribut « voiture qui ne crève pas ». Mais il pourra également percevoir d'autres attributs de valeur liées à cette innovation : puisque la voiture peut rouler à plat, il n'est plus nécessaire de prévoir une roue de secours. Ceci libère de la place dans le véhicule, permet par exemple d'avoir une surface de coffre plane, des sièges sur glissière, un réservoir plus grand, etc. Par ailleurs, le client constructeur est sensible à des attributs de type plus technique, telle que la charge supportée par les pneus, l'insertion dans l'architecture globale du véhicule et de ses performances, etc.

Enfin, il existe un troisième type de client que l'on peut plutôt qualifier de «partie prenante » : la collectivité. Ainsi, si une innovation réduit les émissions de $\mathrm{CO}_{2}$, il y a création de valeur pour la collectivité mais ceci n'est pas une valeur perceptible directement par le

\footnotetext{
${ }^{5}$ Les informations de cette partie proviennent d'une conférence donnée à HEC par trois membres de l'équipe projet de Michelin et de l'interview de l'ancien chef de projet, Monsieur Sortais.

${ }^{6}$ Source : site Internet www. Michelin.fr
} 
client individuel; elle n'est pas non plus perçue comme une valeur par le constructeur automobile tant qu'il n'est pas contraint par une législation à réduire les émissions de $\mathrm{CO}_{2}$.

\section{La mesure de la valeur pour le client final a nécessité des outils marketing sophistiqués}

Les principales étapes de ce travail ont été les suivantes :

- Identifier la liste des attributs de valeur client - les «bénéfices » apportés par l'innovation : sécurité, souplesse, absence de souci, etc.

- Interroger les clients par des méthodes de type analyse conjointe, c'est à dire leur demander d'exprimer leurs préférences sur des ensembles d'attributs qui constituent autant de «voitures types ». En considérant le prix comme un des attributs, ces méthodes permettent également de savoir quelle proportion de clients seraient prêts à payer un certain montant pour se procurer un attribut donné.

- Moduler les résultats obtenus en fonction d'une segmentation des clients. Cette segmentation des clients était fondée sur une analyse sociologique afin de repérer les clients précurseurs, ceux dont les préférences sont susceptibles de se généraliser ultérieurement. Ceci permettait d'avoir une vision dynamique et pas seulement statique de la valeur que l'innovation pourrait apporter sur le marché et d'avoir des prévisions de préférences des clients à un horizon de 10-20 ans.

La dimension temporelle d'un tel projet est effectivement primordiale puisque Michelin doit vendre l'innovation à des constructeurs qui vont l'intégrer dans la définition de leurs nouveaux véhicules. Or le lancement d'un nouveau modèle demande environ 4 ans de développement. Entre le moment où Michelin développe son nouveau pneu et le moment où un client final l'utilisera, il va s'écouler au moins 5 ans. Quant au rachat des pneus par les client au niveau de la rechange, il interviendra encore beaucoup plus tard.

Par ailleurs, l'interrogation des clients a permis incidemment de mettre à jour une demande concernant la détection de pression et donc de proposer des produits qui apportent d'autres bénéfices aux clients. En d'autres termes, l'analyse a révélé des attributs qui n'avaient pas été initialement perçus par l'entreprise.

De plus, l'enquête a révélé que certains « bénéfices » peuvent être considérés comme négatifs pour le client. Ainsi, il est possible qu'un client considère qu'en supprimant la roue de secours, le constructeur cherche à «faire des économies sur son dos ». Il faut donc intégrer cette valeur négative dans l'analyse de la valeur générée par l'innovation. 


\section{Le dialogue avec le client constructeur}

Michelin s'est appuyé sur les bénéfices générés par le nouveau produit tels qu'ils avaient été détectés et valorisés par les clients individuels pour proposer le système PAX aux constructeurs afin que ceux-ci perçoivent à leur tour la valeur créée. Il faut cependant noter les points suivants :

- La valorisation de ces avantages doit être resituée dans le contexte de négociation entre constructeurs et fournisseurs de l'automobile. En particulier, les équipements placés en série dans des véhicules de première monte ne peuvent être perçus comme des avantages distinctifs pour le client. Ils sont donc difficiles à «vendre » pour le constructeur.

- D’une façon plus générale, il est difficile de valoriser une innovation auprès des constructeurs et d'obtenir des prix qui financent les efforts de recherche et développement engagés. Michelin considère que ceci est plus facile en raisonnant en terme de système (incluant le pneu, la roue, l'insert, le capteur de pression et un service crevaison), système dont il garantit la cohérence technique et sur lequel il travaille avec le constructeur lors du développement des futurs véhicules.

- Enfin, dans le bilan économique de l'innovation, il ne faut pas perdre de vue la perte de chiffre d'affaires induite par le fait qu'une voiture ne compte plus que 4 roues au lieu de 5.

\section{La valeur pour le client reste globale}

Même s'il est possible techniquement de décomposer la valeur des attributs par les méthodes d'analyse conjointe, au final, le client porte un jugement global sur un véhicule ; il achète « un tout », pour un prix donné.

\section{LES TENTATIVES DU CONTROLE DE GESTION POUR COUPLER COUTS ET VALEUR}

Nous avons montré dans la première partie de cette communication que la notion de valeur pour le client était imprécise (partie 1.1) mais aussi volatile car dépendante des clients et de leur perceptions (partie 1.2). A supposer que le problème de la définition de la valeur pour le client soit résolu, il faut maintenant voir dans quelle mesure il est possible de relier cette valeur aux coûts qui ont été mis en oeuvre pour la générer afin d'être en mesure de gérer non 
seulement des coûts comme nos «ancêtres du $\mathrm{XX}^{\mathrm{ème}}$ siècle » le faisaient mais des couples coûts-valeur.

McNair et al.(2001) soulignent que le défi de relier la valeur pour le client, le prix et les coûts a été l'objet de nombreuses tentatives autour des nouveaux outils de la comptabilité de gestion (ABC, Target Costing, ABM, TQM, Strategic Cost Management), tentatives qui se sont révélées, selon ces auteurs, «incapables d'évaluer les relations spécifiques qui existent entre les structures de coût internes et une valeur définie de façon externe. »(p. 34). Cependant, McNair et al. apportent assez peu d'arguments pour étayer leur point de vue. De son côté, Gervais (2005, chapitre 11) suggère et étudie quatre outils pour gérer le couple valeur-coût : la gestion par les activités et les processus, l'analyse de la valeur, le coût cible et le feature costing. Pour notre part, nous allons analyser le Target Costing et la méthode $\mathrm{ABC} / \mathrm{ABM}^{7}$ et nous essaierons de voir dans quelle mesure ils peuvent apporter contribuer à expliciter la relation coûts-valeur.

\subsection{LE TARGET COSTING}

La première technique qui vient à l'esprit pour relier le coût d'un produit à sa valeur est celle du coût cible ou target costing puisque telle est précisément sa finalité.

Selon De Rongé, «le coût de revient du produit futur, appelé target cost ou coût cible est déterminé a priori et est la résultante du prix de vente imposé par le marché et du niveau de profit imposé par la stratégie à long terme de l'entreprise » (De Rongé, 2000). Le coût cible ainsi obtenu est ensuite décomposé en utilisant soit la méthode organique - le produit est décomposé en sous-ensembles- soit la méthode fonctionnelle - le produit est décomposé en fonctions, proches de la notions d'attributs. (Lorino, 1994)

Outre le fait que la définition de valeur pour le client retenue par le target costing est explicitement le prix de vente, cette méthode pose deux problèmes de fond qui ont été mentionnés par Lorino dès 1994 :

1. «La description d'un produit comme une combinaison additive de fonctions est-elle toujours réaliste et possible? Un produit n'a-t-il pas une existence systémique par laquelle il serait autre chose que la simple somme de ses parties, même si ces parties sont définies sur une base fonctionnelle plutôt que sur une base technique et organique ? »(Lorino, 1994, p. 40)

\footnotetext{
${ }^{7}$ On peut en effet considérer que l'analyse de la valeur s'intègre dans une démarche de target costing tandis que le feature costing est en fait très proche de l'ABM.
} 
2. «L'hypothèse selon laquelle la structure de coût doit être identique à la structure de valeur est-elle justifiée ?» (Lorino, 1994, p. 41)

Ces deux questions sont reprises par Meysonnier en 2001, ce qui laisse à penser qu'aucune réponse satisfaisante ne leur a été apportée entre ces deux dates : «Deux éléments sont déterminants dans la démarche du target costing. D'abord l'idée que la valeur globale d'un produit pour un client peut être correctement décomposée en attributs indépendants et cumulatifs... Ensuite, le fait qu'il doit y avoir, pour chaque composant du nouveau produit un même niveau d'importance dans le coût de revient que dans la valeur fournie au client : si un composant représente $15 \%$ de la valeur perçue par le client, il doit peser pour $15 \%$ du coût de revient global. » (p. 124)

On notera, avant d'examiner de façon plus détaillée ces deux questions qui sont en fait deux présupposés de la démarche de target costing, que les auteurs cités assimilent la valeur au prix du produit ou utilisent indifféremment ces deux termes, sans se soucier du débat que nous avons soulevé dans la première partie de cette communication.

\section{Premier présupposé: Les attributs seraient des variables indépendantes et se combineraient}

\section{de façon additive pour composer la valeur du produit.}

Pour bien comprendre la nature de ce premier pré-supposé, reprenons l'article d'Horvàth de 1995 qui propose la première illustration d'une application de target costing en français. L'exemple choisi est celui d'un réveil matin. Une étude de marché a été utilisée pour déterminer quelles fonctions les clients recherchaient dans un réveil matin et quel degré d'importance (sur une échelle de 1 à 9) ils accordaient à ces fonctions. Ces degrés d'importance ont ensuite été transformés en pourcentages mais la méthode de conversion n'est pas explicitée ${ }^{8}$; le total des pourcentages de l'ensemble des fonctions est de 100, ce qui laisse supposer que la valeur du réveil matin pour les clients est égal à la somme des valeurs de ses fonctions 9 .

\footnotetext{
${ }^{8}$ Deux méthodes existent pour obtenir ces pourcentages : soit on interroge directement les clients pour leur demander d'affecter des poids aux attributs, soit on déduit les poids de ces attributs par calcul en s'appuyant sur les préférences énoncées par les clients.

9 Horvàrth précise que c'est une méthode d'analyse conjointe qui a été utilisée pour ce faire. L'analyse conjointe permet de traiter des variables dépendantes ou indépendantes. Cependant, les procédures de collecte de données sont beaucoup plus complexes lorsque les variables sont dépendantes et les résultats obtenus doivent tenir compte de ces relations entre variables. Dans le cas précis, Horvàrth ne précise pas le type d'analyse conjointe qui a été utilisée ; on peut donc faire l'hypothèse qu'il a considéré les variables comme indépendantes, cas le plus simple et le plus courant.
} 
Or ce postulat est contredit par l'intuition, par la comparaison avec les processus industriels et par le développement de concepts de marketing, en particulier ceux appliqués aux services.

a) Intuitivement, rien ne permet d'affirmer que les attributs sont indépendants. En particulier, on peut penser que le design d'un réveil n'aura de valeur pour le client que si une fonction élémentaire comme l'exactitude est remplie correctement. Il n'y aurait donc pas indépendance des attributs et pour cette raison, il n’y aurait pas non plus d'additivité des attributs. Par ailleurs, comment un modèle de type additif peut-il gérer le fait que certains attributs peuvent être «contradictoires»? Par exemple, l'accroissement de la sécurité d'une voiture par l'ajout d'airbags et de portes renforcées augmente la consommation de carburant.

b) La comparaison avec les notions issues de la qualité en environnement industriel permet d'éclairer et de renforcer ce point : dans les industries d'assemblage (comme l'électronique), la fiabilité d'un produit fini dépend de la fiabilité des composants mais de façon multiplicative. En effet, si 99 pièces sont correctes mais que la centième est défectueuse, le niveau de qualité ne sera pas de $99 \%$ mais de $0 \%$ (l'appareil ne fonctionnera pas); de la même façon, dans la gestion des services, il suffit parfois d'un détail défectueux (par exemple une ampoule grillée au dessus du lavabo dans une chambre d'hôtel) pour modifier la perception de qualité que le client aura d'un service par ailleurs satisfaisant. Dans l'industrie, on distingue d'ailleurs les défauts critiques des autres : ce sont ceux qui ont un impact décisif sur la qualité réalisée ou perçue du produit.

c) Dans le domaine du marketing, Naumann et Jackson (1999) ont formalisé une distinction s'inspirant des ressources humaines entre les facteurs d'hygiène (ceux pour lesquels le fournisseur doit atteindre un minimum de qualité requis sous peine d'entraîner l'insatisfaction) et les facteurs de satisfaction, qui comme leur nom l'indique, génèrent des perceptions positives. Pour un même attribut, Parasuraman et al. (1991) ont d'ailleurs montré que les attentes des clients n'étaient pas linéaires et qu'elles présentaient des effets de seuil, la plupart des clients distinguant un niveau «adéquat» en dessous duquel les clients vont porter un jugement négatif sur le service et un niveau de service « désiré » au delà duquel les clients vont porter un jugement positif (l'intervalle entre le niveau adéquat et le niveau désiré constituant une zone de tolérance). 
Les attributs ne jouent donc pas tous le même rôle : certains peuvent être éliminatoires (comme une note dans une matière à un examen), certains ne jouent que s'ils sont affectés d'une note négative, d'autres encore impactent la valeur globale dans tous les cas de figure (Loosa, 1997). La valeur d'un produit à partir de la valeur de ses attributs ne peut pas se calculer comme la moyenne des notes du baccalauréat.

Dès 1994, Lorino mentionne que l'analyse fonctionnelle s'est efforcée d'apporter une réponse à ce problème en distinguant les «fonctions exigées » liées à des performances techniques et à l'usage même de l'objet, des «fonctions valorisables » ou fonctions de prestige liées à la satisfaction de besoins plus flous et portées plus globalement par l'entité - produit dans son intégralité. Toutefois, les solutions techniques évoquées par Lorino dans cet article ne sont en général pas reprises et approfondies dans les manuels de comptabilité de gestion les plus récents (Demeestère, 2004, Bouquin, 2004). D'ailleurs, en 2001, Meyssonier, s'appuyant sur l'exemple de Renault, souligne à son tour la nécessité de préserver «l'identité globale du produit, même si cela est parfois en contradiction avec la logique de décomposition et d'additivité des attributs. »(p. 125) et Mévellec (2005) conteste également que la valeur globale soit égale à la somme des valeurs des attributs : "Pour autant, il est difficile de prétendre que la valeur globale est la somme des valeurs unitaires de ces fonctionnalités. » (p. 57)

Différentes modalités de mise en oeuvre de l'analyse conjointe existent pour appréhender la complexité de la hiérarchie des attributs. Ainsi, l'analyse conjointe peut traiter des variables dépendantes (c'est-à-dire des attributs qui sont liés les uns aux autres), elle peut traiter des préférences non linéaires (effets de seuil par exemple). Moyennant quelques transpositions de calcul, il est possible de remplacer des préférences additives par des préférences multiplicatives. Mais pour parvenir à ce niveau de finesse, il faut naturellement accroître le coût et la complexité de la collecte de données et les résultats obtenus sont plus difficiles à lire et à interpréter. Enfin, l'analyse conjointe peut éventuellement donner la valeur d'un attribut (par exemple le montant qu'un client est prêt à payer pour avoir des champignons sur sa pizza en plus des autres éléments de garniture) mais il s'agit d'une valeur marginale, déterminée attribut par attribut, le reste de la définition du produit étant figé. La somme de ces valeurs marginales ne donne pas la valeur totale du produit (dans notre exemple, la pâte génère un coût mais n'a pas de valeur marginale pour le client car on ne peut pas imaginer une pizza sans pâte). 
Enfin, l'analyse conjointe et d'une façon plus générale la décomposition de la valeur globale d'un produit en « sous valeurs » par attribut ne fonctionne bien qu'à deux conditions :

- D’une part que le produit puisse être effectivement décomposé en attributs, ce qui n'est pas nécessairement le cas avec des produits que le client appréhende comme une « expérience globale », par exemple un film (Aurier et al., 2004).

- D'autre part que le client ait une consommation suffisamment fréquente et répétée ou qu'il puisse recueillir des informations ex ante pour pouvoir construire son jugement. Cette condition peut être difficile à satisfaire, en particulier pour les services sur lesquels le client ne se forge une opinion qu'après les avoir expérimentés.

Comme nous venons de le voir, il est donc possible d'avoir recours à des méthodes qui ne reposent pas sur le premier présupposé pour établir une relation entre un attribut et une valeur mais ces méthodes sont complexes et lourdes à mettre en œuvre.

\section{Deuxième présupposé : L'optimum dans la gestion des coûts et de la valeur serait obtenu en respectant la proportionnalité entre coûts et valeur.}

Ce second présupposé apparaît de façon encore plus claire que le précédent dans l'article de Horvàrth (1995) puisqu'il écrit: «Le but du target costing est que chaque composant engendre un montant de coûts correspondant à la valeur que lui attribue le consommateur » (p. 76-77). Ce propos est renforcé par la figure 9 (p. 78) qui montre une «zone de valeur optimale » dans laquelle la proportionnalité entre coûts et valeur est respectée.

Il est impossible de trouver un raisonnement sous-tendant cette position : «On ne voit pas au nom de quoi les deux devraient coïncider. Et on imagine facilement les dérives possibles : l'application d'un tel principe peut, par exemple, pousser à un gaspillage inconsidéré au niveau d'un attribut du produit simplement pour respecter une adéquation entre coût et valeur alors qu'un coût inférieur serait possible. » (Meyssonier, 2001, p. 125). Rien n'empêche en effet une fonction à très forte valeur de pouvoir être réalisée pour un coût très faible... ou une fonction indispensable mais avec une valeur limitée de coûter très cher à produire.

L'information produite par une démarche de Target Costing pour gérer les coûts pourrait donc être utilisée de deux manières :

- De façon globale pour vérifier l'adéquation entre le coût prévisionnel d'un produit et son futur prix de marché ; 
- De façon plus fine, au niveau des composants ou des fonctions du produit, mais en sachant que, dans ce cas, les valeurs de ces composants ou fonctions ne constituent que des signaux pour fixer des objectifs de coût et certainement pas une norme à respecter envers et contre tout.

\subsection{L'APPROCHE PAR LES ACTIVITES ET PROCESSUS}

La comptabilité d'activités a fait émerger les notions d'activités et de processus comme centrales pour faciliter le calcul des coûts. Rapidement, les différents auteurs ou utilisateurs des méthodes de type $\mathrm{ABC}$ se sont aperçus qu'ils pouvaient également utiliser les activités comme des supports au management. Finalement, certains estiment qu'il est possible de relier les coûts à la valeur créée pour le client au travers de ces activités et processus (et non plus au niveau des produits comme dans le target costing). Cette vision est particulièrement nette dans l'article de Chauvey et Naro (2004) qui expliquent que trois modèles de causalité sont possibles : celui qui lie les activités aux coûts, celui qui lie les activités à la valeur et celui qui lie la valeur et les coûts, «la maîtrise de cette dernière relation devant garantir à l'entreprise un avantage concurrentiel ». Nous allons étudier dans cette partie l'ensemble des travaux qui s'appuient sur les activités et les processus pour relier coûts et valeur.

\subsubsection{Les propositions de Lebas et Mévellec}

Au travers d'une série d'articles (Lebas, 1991, Lebas et Mévellec, 1999, Mévellec, 2000a et 2000b), Lebas et Mévellec s'efforcent de montrer qu'un système de calcul des coûts a deux fonctions : gérer les coûts et simuler la valeur créée pour le client. En même temps, ils énoncent les conditions à remplir pour que les systèmes utilisés par les entreprises puissent effectivement remplir ces deux fonctions. Nous allons repartir de l'article de Lebas de 1991 qui présente l'avantage de définir la notion d'activité à valeur ajoutée, puis nous examinerons l'article 2000b de Mévellec qui fait apparaître clairement une chaîne de valeur constituée d'activités rassemblées dans un processus au sein de l'entreprise. Enfin, nous analyserons simultanément l'article co-écrit en 1999 et celui publié par Mévellec seul en 2000 (2000a) car leurs contenus sont très proches.

Après avoir découpé l'entreprise en activités, et après avoir calculé un coût par activités, Lebas (1991) propose de distinguer les activités à valeur ajoutée et les activité sans valeur ajoutée. Il utilise la définition suivante : «Une activité est à valeur ajoutée si sa réalisation augmente l'intérêt du client pour notre produit ou notre service. » (p. 58). Lebas se place ainsi dans une perspective de valeur-utilité et se rapproche de la définition de la valeur comme 
utilité/satisfaction pour le client que nous avons retenue. Il ajoute que sa définition est issue de Porter (1985) qui a « un concept de valeur ajoutée positif et volontariste; il est défini à partir du client en fonction de la perception qu'en a l'entreprise. » (p. 58)

Par ailleurs, Lebas précise que la décision d'affecter une activité à l'une ou l'autre catégorie (avec ou sans valeur ajoutée) est de nature stratégique. En effet, on comprend très bien qu'une activité sans valeur ajoutée pour une entreprise (par exemple des contrôles qualité) peut être à valeur ajoutée pour une autre entreprise (par exemple si elle est sous-traitante dans le secteur nucléaire). Toutefois, il faudrait sans doute aller plus loin dans la distinction entre activités à valeur ajoutée et sans valeur ajoutée et dans la gestion des conséquences opérationnelles de cette classification. En effet, les entreprises ont souvent plusieurs segments de clientèle, qui ont des exigences hétérogènes. Que faire des activités qui sont à valeur ajoutée pour certains segments et sans valeur ajoutée pour d'autres? De plus, les exemples d'activités avec ou sans valeur ajoutée donnés par Lebas laissent un peu perplexes. Ainsi, le nettoyage industriel « contribue éventuellement à la qualité des produits donc peut être considéré comme étant à valeur ajoutée » (p. 59-60). Mais que dire des cas où le nettoyage industriel intervient parce que le travail est exécuté dans de mauvaises conditions d'organisation et de propreté ? Faut-il décomposer l'activité de nettoyage en deux catégories ? Sur quelles bases ? En bref, déclarer une activité avec ou sans valeur ajoutée, même dans une perspective stratégique, n'est-ce pas déjà un «jugement de valeur »?

Enfin, dans cet article de 1991 de Lebas, il manque encore un lien entre l'activité et le produit ou le client, lien qui va être introduit ultérieurement grâce au concept de processus qui apparaît clairement dans l'article de Mévellec sur la comptabilité d'activités (Mévellec, 2000b).

Pour Mévellec, une fois la liste des activités établies, il faut établir la carte des activités, c'està-dire les regrouper au sein de processus. C'est la constitution de ces processus qui donne sens à l'ensemble de la démarche. Les processus doivent en effet recréer une chaîne de la valeur, c'est à dire « regrouper les activités qui forment, de par leur relation, un segment de la chaîne de valeur de l'entreprise. Ces activités concourent toutes à la délivrance d'un attribut porteur de valeur pour le client » (p. 400). L'auteur précise : «C'est à ce stade que l'on essaie de prendre en considération la nécessité de gérer le coût et la valeur ». Et, de fait, si l'on dispose du coût par activités et que ces activités sont regroupées en des processus qui génèrent de la valeur pour le client, il sera possible de gérer, au travers d'une seule modélisation, les 
coûts et la valeur. Le dispositif proposé par Mévellec dans cet article appelle cependant quelques remarques.

- Tout d'abord, la vision de la valeur utilisée par Mévellec est une vision interne, construite au sein de l'entreprise. Il parle d'ailleurs à plusieurs reprises de simulation de la valeur et non pas de mesure de la valeur. Pour nous, si la valeur vient de la perception que le client a des attributs du produits/service, elle ne peut se calculer ou se simuler de façon interne, elle doit émaner de données collectées sur les marchés, auprès des clients. De façon troublante, Mévellec assimile parfois la simulation de la valeur au calcul du coût : « ...pour simuler la valeur (calculer le coût) » (p. 404). En fait, c'est uniquement à la fin de son article de 2000b que Mévellec introduit les perceptions du client au travers d'un exemple qui suggère que le coût calculé doit être différent si un client accorde plus d'importance à un attribut qu'un autre client. Ce type d'ajustement est qualifié de «réglage du système ». Il n'est pas au cœur de la démarche proposée.

- D'ailleurs, les exemples de processus donnés par Mévellec ressemblent à des processus de transformation tel qu'on les voit dans des analyses de production ou de gestion de la qualité. Il semblent «poussés » par les opérations effectuées plus que « tirés » par le marché ou les clients et rien ne permet d'affirmer que cet ensemble d'activités/opérations va créer de la valeur perçue par les clients.

- Enfin, Mévellec considère qu'il peut y avoir une bijection entre les processus et les attributs : par construction, un processus mène en principe à un attribut. Mais ne peut-il y avoir des attributs qui mobilisent des activités issues de plusieurs processus ${ }^{10}$ ? Ou, à l'opposé, des activités qui concourent à la réalisation de plusieurs attributs? Dans son schéma de l'architecture générale du modèle (figure 2 page 401), Mévellec ne fait d'ailleurs pas apparaître le lien entre les processus et les attributs ; la façon dont les processus et les attributs s'articulent reste imprécise.

A l'opposé, dans les articles de Lebas et Mévellec (1999) et de Mévellec 2000a, on a parfois l'impression que la valeur est dictée par le marché et non construite (simulée) au sein de l'entreprise : «Ce qui intervient ensuite, le calcul du coût unitaire des produits, relève d'une autre démarche. Il s'agit maintenant de confronter la consommation des ressources telle qu'elle vient d'être décrite, avec la valeur que les clients vont accepter de payer» (Mévellec,

\footnotetext{
${ }^{10}$ Lorino évoque aussi le fait qu'un même attribut (une même fonction) peut être réalisé par des moyens très différents selon le client qui le réclame et la situation dans laquelle il se trouve (Lorino, 1995, p. 68)
} 
2000a, p. 33). Dans ces deux articles, les auteurs s'appuient sur une analyse historique des systèmes de calcul des coûts. Ils distinguent la période traditionnelle, durant laquelle la valeur est assimilée à la somme des coûts (en particulier des coûts de main d'œuvre) et la période récente, au cours de laquelle la vision qui s'impose est que «la valeur est le résultat des attributs perçus du produit » (1999, p. 16). Cette opposition chronologique ne va cependant pas de soi car les auteurs mentionnent que, à partir des années 50 aux Etats-Unis, « la valeur est déterminée sur le marché » (Mévellec 2000, p. 35). Ceci aurait entraîné la naissance des systèmes en coûts partiels.

S'appuyant sur cette idée de valeur émanant du marché ${ }^{11}$, les auteurs proposent donc de partir des attributs de valeur perçus par les clients, d'identifier les générateurs de valeur qui les expliquent et de déterminer les coûts attachés à ces générateurs pour avoir le coût des attributs (1999, figure 2, p. 17). Cette méthode permettrait donc effectivement de relier le coût et la valeur. Malheureusement, des contradictions demeurent dans ces deux derniers articles (la figure 2 elle même a pour titre : mécanisme de simulation de la valeur !) et les méthodes permettant d'isoler les générateurs de valeur puis de les relier aux activités pour avoir leur coûts ne sont pas expliquées. Enfin, le problème de la décomposition de la valeur globale en valeur des attributs n'est pas abordé dans ces textes.

\subsubsection{La proposition de McNair-Polutnik-Silvi}

Après avoir montré rapidement les limites des outils de comptabilité de gestion destinés à comprendre les relations entre coût et valeur, McNair et al. (2001) proposent un modèle intitulé «Value Creation Model » (VCM) destiné à combler ce manque. Nous allons d'abord décrire la méthode telle qu'elle apparaît dans leur article, puis nous la commenterons en montrant ses limites.

\section{Description de la méthode :}

1. Pour chaque segment de marché, on crée un profil type de client avec une liste d'attributs et leurs poids respectifs, tels qu'ils sont perçus par les clients; le total des poids des attributs est de 100 .

\footnotetext{
${ }^{11}$ Notons cependant pour mémoire que, dans son livre de 2005, Mévellec ne retient finalement aucune de ces deux options -valeur calculée ou valeur dictée par le marché- puisqu'il écrit : «La valeur n'est ni dictée par un coût interne ni déduite du fonctionnement du marché mais co-construite dans l'échange lui-même » (p. 57)
} 
2. Pour chaque segment de marché, on calcule une valeur approchée des attributs en multipliant le chiffre d'affaires réalisé sur le segment par le poids affecté à l'attribut.

3. Une analyse des activités réalisées par chaque Département est menée. Les coûts des activités sont recensées. Puis, les activités et leurs coûts sont ventilés en trois catégories: les activités à valeur ajoutée, les activités sans valeur ajoutée mais nécessaires et le gaspillage.

4. L'étape suivante proposée dans l'article montre la ventilation des coûts par attribut de segment de marché, ce qui permet de comparer le coût d'un attribut et son poids pour le client. Les coûts par attributs sont également ventilés en coûts à valeur ajoutée, sans valeur ajoutée et en gaspillage.

\section{Remarques :}

- Les deux premières étapes sont similaires à la démarche de target costing si ce n'est que l'on remplace le prix de vente du produit par un chiffre d'affaires et que l'on travaille par segment de clientèle. De ce fait, on se heurte à nouveau aux présupposés portant sur l'indépendance et l'additivité des attributs.

- La troisième étape constitue le début d'une démarche $A B C$ et le classement des activités en trois catégories est similaire à celui de Lebas encore que l'on puisse se poser des questions sur la catégorie des activités sans valeur ajoutée mais nécessaires : Nécessaires à qui ?

- Aucune précision n'est donnée dans l'article sur la façon dont la quatrième étape se déroule. En particulier, le lecteur ne sait pas comment et par quel mécanisme ou raisonnement les coûts des activités sont reliés et ventilés sur les attributs. Ceci est d'autant plus ennuyeux que l'on peut imaginer qu'un attribut mobilise plusieurs activités. Selon nous, le « chaînon manquant » entre coûts et valeur manque toujours à l'appel... De plus, le rôle de la décomposition entre les trois types d'activités n'apparait pas clairement. De ce fait, cette distinction, par ailleurs discutable au vu des exemples donnés, ${ }^{12}$ complique la démonstration sans l'enrichir. Enfin, à supposer que le problème de la relation entre les coûts des activités et la valeur des attributs ait été résolu, les auteurs adoptent le même postulat que le target costing, à savoir qu'il

\footnotetext{
${ }^{12}$ Les départements approvisionnement et logistique ne comptent aucune activité à valeur ajoutée, les visites au client sont classées dans les activités de gaspillage, de même que la surcapacité, etc.
} 
faut harmoniser le poids des attributs et leurs coûts pour améliorer les performances de l'entreprise.

\subsubsection{La proposition de Lorino}

La position adoptée par Lorino concernant la nécessité d'établir une relation entre coûts et valeur est beaucoup plus nuancée que celles des auteurs cités précédemment. D’un côté, il considère que cette mise en relation est indispensable, qu'elle seule doit intéresser le gestionnaire et permettre de porter un jugement sur la performance de l'entreprise : «En fait, la seule question économique pertinente pour le pilotage de l'entreprise est l'optimisation du couple coûts fonctionnalités (coût/valeur) » (Lorino, 1995b, p. 130). D'un autre côté, il insiste abondamment, tant dans son ouvrage «Comptes et Récits de la Performance » que dans l'article paru la même année (1995), «Le déploiement de la valeur par les processus » sur les difficultés qu'il y a à établir cette relation et à la décliner au sein du réseau d'activités d'une entreprise.

Pour Lorino, la mise en relation des coûts et de la valeur créée se heurte à deux difficultés : d'une part, il y a un décalage dans le temps entre des coûts qui sont immédiats et une valeur qui ne se produira nécessairement que plus tard, lorsque le client achètera le produit (Lorino, 1989, p. 155); d'autre part, du fait de l'organisation du travail en entreprise, une activité correspond rarement à une fonctionnalité attendue par le client : « Il faut en général combiner plusieurs activités de manière souvent complexe pour produire une fonction et une activité donnée contribue à l'obtention de plusieurs fonctions distinctes » (1995a, p. 59). Ainsi, pour Lorino, «c'est la nature sociale (division du travail) et diachronique (étalement dans le temps) de la création de valeur qui rend la correspondance fonction/activité problématique. » (1999a p. 59)

Pour effectuer un pilotage des activités qui tienne compte de la valeur créée, il faut donc connaître la genèse de la valeur au travers des activités, connaissance qualifiée de problématique par Lorino (1995b, p. 132) du fait qu'il considère qu'il s'agit plutôt d'une interprétation : "Comment définir des règles de conduite sur des activités en fonction de leur contribution, difficilement modélisable, à une interprétation-valeur future aux modalités incertaines? Il n'y a évidemment pas de réponse mathématique, algorithmique, parfaitement rationnelle à cette question. Y répondre est affaire de jugement, d'interprétation, à travers des enchaînements causes-effets multiples, flous, complexes. » (p. 133) 
Toutefois, Lorino considère que les processus peuvent pour partie résoudre ce problème, car ces derniers vont structurer les activités en fonction du résultat final, des clients et des produits obtenus : «Le processus est un passage obligé entre activités et fonctions, entre coûts et valeur, ce passage fut-il tortueux et contourné. » (1995a, p. 60). «Par la construction de processus, l'entreprise essaie de surmonter le péché originel qu'est la division du travail et la perte de contact avec le jugement de valeur futur (avec le client) qui en découle pour la majeure partie de la population de l'entreprise. Il s'agit donc de contrer la fragmentation en activités spécialisées en procédant à des agencements d'activités signifiants du point de vue de la création de valeur (réponse aux besoins d'un client). » (1995 a, p. 62)

Parallèlement, Lorino attribue des montants de ressources aux processus. Il divise ces ressources en trois catégories : celles utilisées pour créer de la valeur, celles utilisées mais qui ne créent pas de valeur et celles qui ne sont pas utilisées (surcapacité) mais qui pourraient créer de la valeur par exemple en accroissant la flexibilité du processus de production et en améliorant la réponse aux demandes des clients.

Si l'on compare les travaux de Lorino et ceux de Lebas et Mévellec, on constate donc des points communs mais aussi des différences notoires :

- Pour Lorino, la valeur trouve son origine dans le jugement du client. Il faut certes anticiper ce jugement, mais la valeur n'est pas construite, simulée ou calculée comme le laissent supposer certains écrits de Mévellec.

- La valeur se fonde sur des fonctionnalités pour Lorino et sur des attributs pour Lebas et Mévellec mais cette nuance de vocabulaire ne semble pas induire de différences majeures.

- Lorino propose, comme Mévellec, de construire des processus à partir des activités, pour faire apparaître des chaînes de création de valeur. Toutefois, Lorino est très réservé sur les possibilités de relier de façon systématique les attributs aux activités qui les génèrent tandis que Lebas et Mévellec suggèrent d'inclure ces relations dans le modèle de représentation des activités, et même de s'en servir pour structurer le modèle.

- En fait, la perspective retenue par Lorino est moins comptable qu'organisationnelle. Il cherche davantage à gérer les processus qu'à établir un modèle comptable ou financier reliant explicitement les coûts et la valeur. 


\section{CONCLUSION}

Ainsi, il apparaît que la gestion conjointe des coûts et de la valeur créée pour le client passe par un certain nombre d'étapes qui posent problème.

D'une part, il faut définir la valeur globale pour le client et les avis divergent pour savoir si celle-ci est une synthèse entre le prix et les fonctionnalités du produit ou bien si la valeur ne se construit qu'à partir des bénéfices que le client va retirer de l'usage d'un produit, le prix pouvant alors être considéré comme un indicateur de la valeur.

Ensuite, la valeur globale d'un produit s'expliquant par les attributs qui caractérisent ce produit, il faut estimer la valeur de chacun des attributs pris isolément ; cette tâche présente un certain nombre de difficultés techniques et à la fin de ce travail, il n'est pas sûr que la somme des valeurs des attributs soit égale à la valeur globale du produit.

L'étape suivante consiste alors à calculer les coûts générés par la production des attributs. Pour ce faire, les spécialistes de comptabilité de gestion préconisent de s'appuyer sur une modélisation de l'organisation en activités et processus. Dans l'idéal, ce découpage en activités et processus devrait se faire de telle sorte que chaque processus concoure à produire un attribut pour que l'on puisse isoler les coûts et les rattacher aux attributs ; dans la pratique cependant, étant donnée la complexité des processus de travail dans les grandes entreprises, il ne sera pas toujours possible d'isoler strictement les activités qui contribuent à la réalisation d'un attribut.

Enfin, il reste à appréhender la dimension temporelle du problème, les coûts précédant la valeur dans le temps, et ce parfois de plusieurs mois. De ce fait, les prises de décisions s'appuyant sur une comparaison entre coûts et valeur agrègent des éléments qui ne se situent pas dans le même horizon temporel.

La construction d'une modélisation de l'entreprise qui permettrait de relier coûts et valeur pour le client apparaît donc comme une tâche extrêmement complexe. De plus, la valeur étant amenée à changer dans le temps et pour les différentes catégories de clients, si l'on suppose que l'on opère les mises à jour nécessaires, les résultats produits par le modèle feraient preuve d'une certaine volatilité qui les rend difficiles à utiliser pour prendre des décisions de moyen ou long terme.

Toutefois, puisque les études réalisées dans le domaine du marketing permettent d'approcher la notion de valeur des attributs pour le client, il serait dommage de se priver de ces informations pour orienter les décisions qui pourront être prises sur les processus, les activités, le développement des produits. Nous suggérons cependant que cette utilisation se 
fasse de façon ponctuelle, dans des cas ou sur des dossiers précis, la modélisation globale du couple coûts-valeur se heurtant à la complexité de la notion de valeur et à celle des processus qui créent cette valeur. 


\section{BIBLIOGRAPHIE}

P. Aurier, Y. Evrard, G. N'Goala (2004) : «Comprendre et mesurer la valeur du point de vue du consommateur », Recherche et Applications en Marketing, Vol. 19, n 3/2004, p. 1-20.

P. Barwise et S. Meehan, (1999), « De l'importance de la perception de valeur par le client », L'Art du Marketing, Les Echos, 16-17 avril.

H. Bouquin (2004), Comptabilité de gestion, Economica.

A. Bourguignon, (2005), «Management Accounting and Value Creation: the Profit and Loss of Reification », Critical Perspectives on Accounting, Vol. $\mathrm{n}^{\circ}$ 16, p. 353-389.

J.P. Bréchet et A. Desrumeaux, (2001), «Le thème de la valeur en sciences de gestion représentations et paradoxes », Revue Sciences de Gestion, n ${ }^{\circ} 28$, p. 217-245.

M. Bromwich, (1990), «The Case for Strategic Management Accounting: the Role of Accounting Information for Strategy in Competitive Markets », Accounting, Organizations and Society, Vol. $15 \mathrm{n}^{\circ} 1 / 2$, p. 27-46.

Z.Carmon et D. Ariely (2000) : "Focusing on the Forgone: How Value Can Appear So Different to Buyers and Sellers", Journal of Consumer Research, Vol. 27, December, p. 360370.

G. Charreaux et P. Desbrières, (1998), «Gouvernance des entreprises : valeur partenariale contre valeur actionnariale », Finance Contrôle Stratégie, Vol. 1, n 2, juin, p. 57-88.

J.N. Chauvey et G. Naro, (2004), «Les apports de l'ABC à l'analyse stratégique : les enseignements d'une recherche-intervention », Finance Contrôle Stratégie, Vol 7, n 3, p. 6389.

R. Demeestère (2004), Comptabilité de gestion et mesure des performances, Dunod.

Y. De Rongé, (2000), «Coûts », in Encyclopédie de Comptabilité, Contrôle de Gestion et Audit, sous la direction de B. Colasse, Economica.

B. Dubois, (1996), «L'ère du marketing situationnel », Marketing Magazine, $\mathrm{n}^{\circ}$ 10, mars, p. 12-13.

B. Dubois, (1997), «Le marketing situationnel va devenir stratégique», Marketing Magazine, $\mathrm{n}^{\circ} 26$, décembre, p. 42-44.

C.D. Echaudemaison, (1989), Dictionnaire d'économie et de sciences sociales, Nathan.

J. Gadrey, (1988) : «Productivité, output médiat et immédiat des activités de service : les difficultés de transfert d'un concept », in L'Europe face à la nouvelle économie de service, PUF, p.113-141.

M. Gervais, (2005), Contrôle de gestion, Economica, $8^{\text {ème }}$ édition. 
P. Horvàth, (1995), « Pour un contrôle de gestion à l'écoute du marché », Revue Française de Gestion, juin-juillet-août, p. 72-85.

C.D. Ittner et D.F. Larcker, (1998), « Innovations in Performance Measurement: Trends and Research Implications", Journal of Management Accounting Research, vol. 10, p. 205-238.

F. Jallat, (2002) : «Le client, meilleur allié de l'actionnaire », L'Expansion Management Review, juin, p. 68- 74.

G. Kœnig (2004) : "Management stratégique - projets, interactions \& contextes”, Dunod.

M. Lebas, (1991), «Comptabilité analytique basée sur les activité, analyse et gestion des activités », Revue Française de Comptabilité, n 226, septembre, p. 47-63.

M. Lebas et P. Mévellec (1999), «Simultaneously Managing Cost and Value: The Challenge", in "The Role of Management Accounting in Creating Value", IFAC.

S. Loosa (1997), "L'analyse de la contribution des éléments du service à la satisfaction : un modèle tétraclasse », Décisions Marketing, $\mathrm{n}^{\circ}$ 10, janvier-avril, p. 81-88.

P. Lorino, (1994), «Target Costing ou gestion par coût cible», Première partie, Revue Française de Comptabilité, ${ }^{\circ} 255$, p. 35-45.

P. Lorino, (1995a), «Le déploiement de la valeur par les processus », Revue Française de Gestion, juin-juillet-août, p. 55-71.

P. Lorino, ( 1995b), Comptes et récits de la performance, Les Editions d'Organisation.

P. Lorino, (1997), Méthodes et pratiques de la performance. Le guide du pilotage, Editions d'Organisation.

C.J. McNair, L. Polutnik, R. Silvi (2001), «Cost Management and Value Creation: the Missing Link », The European Accounting Review, Vol. 10, n 1, p. 33-50.

P. Mévellec ( 2000a), « Lecture duale des systèmes de coûts : bilan d'étape d'une démarche de recherche de formation- action », Comptabilité Contrôle Audit, mars, p. 27-46.

P. Mévellec (2000b), «Comptabilité par activités», in Encyclopédie de Comptabilité, Contrôle de Gestion et Audit, sous la direction de B. Colasse, Economica, p. 395-405.

P. Mévellec (2005), Les systèmes de coûts, Dunod, Paris.

F. Meysonnier (2001), «Le target costing: un état de l'art», Finance Contrôle Stratégie, Vol. 4, n 4, décembre, p. 113-138.

E. Naumann et D. Jackson (1999), «One More Time: How Do You Satisfy Customers ?», Business Horizons, May-June, p. 71-76.

A. Parasuraman, L. Berry, V. Zeithaml (1991), "Understanding Customer Expectations of Service", Sloan Management Review, Spring, p. 39-48. 
M. Porter, (1985), Competitive advantage, Free Press, New-York

R. Ramirez, (1999), «Value Co-Production: Intellectual Origins and Implications for Practice and Research », Strategic Management Journal, n 20, p. 49-65.

L. Roche, (2002), «La valeur, une notion sans cesse réinventée », L'Expansion Management Review, juin, p. 32- 40.

C. Simon (2000), "Valeur et comptabilité », in Encyclopédie de Comptabilité, Contrôle de Gestion et Audit, sous la direction de B. Colasse, Economica.

A.Smith, (1991), Recherche sur la nature et les causes de la richesse des nations, Gallimard Folio.

K. Snellman et T. Vihtkari, (2003), « Customer complaining behaviour in technology-based service encounters », International Journal of Service Industry Management, vol. 14, ${ }^{\circ}$ 2, p. 217-231.

R. Teller, (1999), Le contrôle de gestion - Pour un pilotage intégrant stratégie et finance, Editions Management \& Société. 